\title{
Significant Improvement of Mechanical Properties for Polyvinyl Alcohol Film Prepared from Freeze/Thaw Cycled Gel
}

\author{
Taishi Fukumori, Takahiko Nakaoki* \\ Department of Materials Chemistry, Ryukoku University, Otsu, Japan \\ Email: *nakaoki@rins.ryukoku.ac.jp
}

Received July 25, 2013; revised August 25, 2013; accepted September 1, 2013

Copyright (C) 2013 Taishi Fukumori, Takahiko Nakaoki. This is an open access article distributed under the Creative Commons Attribution License, which permits unrestricted use, distribution, and reproduction in any medium, provided the original work is properly cited.

\begin{abstract}
The mechanical properties of polyvinyl alcohol (PVA) films prepared by evaporating water from freeze/thaw cycled gel were investigated as a function of the number of freeze/thaw cycles. The maximum stress of the PVA film prepared by freeze/thaw cycling was larger than that prepared without the freeze/thaw cycle process. The largest maximum stress was 46.2 MPa for a film prepared with 10 freeze/thaw cycles, which was twice as large as that for a cast PVA film without freeze/thaw cycling $(22.3 \mathrm{MPa})$. This is due to the formation of small crystallites during the freeze/thaw cycle process. Furthermore, when the film was annealed at $130^{\circ} \mathrm{C}$, the maximum stress was as high as $181 \mathrm{MPa}$ which was comparable to that for PVA films prepared using additives. The crystallinity is not the main factor that determines the maximum stress for either the non-annealed or annealed freeze/thaw cycled films, but the glass transition temperature is well correlated with the maximum stress, irrespective of the annealing process. This is due to the different molecular morphology; the non-annealed freeze/thaw cycled film consists of many small crystallites, but the annealed film consists of larger crystallites formed during the annealing process.
\end{abstract}

Keywords: Polyvinyl Alcohol; Mechanical Properties; Freeze/Thaw Cycle; Film

\section{Introduction}

Polyvinyl alcohol (PVA) is a representative water soluble polymer that is widely investigated as a host material for nanocomposites. There have been many reports on the mechanical properties of PVA films with additives. The mechanical properties are strongly dependent on the degree of saponification and the maximum stress for PVA film with saponification of ca. $98 \%$ is improved to ca. 60 MPa (original PVA: ca. $20 \mathrm{MPa}$ ) [1], $72 \mathrm{MPa}(28 \mathrm{MPa})$ [2], and $42 \mathrm{MPa}(17 \mathrm{MPa})$ [3] by the addition of $\mathrm{Ag}$, aminoclay plus Ag, and graphene, respectively. PVA film with saponification of $>99 \%$ provided higher maximum stress, such as 83 and $95 \mathrm{MPa}$ reported by Zang et al. [4] and Morimune et al. [5], respectively, and further improvement to 148 and $124 \mathrm{MPa}$ was achieved by the addition of polyvinylpyrolidone (PVP)/sodium dodecyl sulfate (SDS)/single wall carbon nanotubes (SWNTs) and nanodiamond, respectively. Significant improvement of the mechanical properties of PVA film was also re-

${ }^{*}$ Corresponding author. cently reported using $\mathrm{Na}^{+}$-montmorillonite $+\mathrm{Cu}^{2+}$ and nanodiamond + SWNTs of which the maximum stresses were as high as ca. $320 \mathrm{MPa}$ (original PVA: $40 \mathrm{MPa}$ ) [6] and 534.3 $\mathrm{MPa}(38.0 \mathrm{MPa})$ [7], respectively. Annealing is another factor used to improve the mechanical properties of PVA. PVA with saponification of $98 \%-99 \%$ that was annealed at $130^{\circ} \mathrm{C}$ exhibited a maximum stress as high as $137 \mathrm{MPa}$ [8]. These results indicate that regulation of the morphology and crystallinity is important factors in improving the mechanical properties of PVA film.

PVA forms a stable hydrogel by physical cross-linking. It has been reported that repeated cycling processes of freezing at $-20^{\circ} \mathrm{C}$ and thawing at room temperature provides a thermoreversible hydrogel $[9,10]$. PVA hydrogel prepared by freeze/thaw cycles has attractive properties, such as good mechanical properties, rubber-like flexibility, and high water content. The molecular structure of this PVA hydrogel is characterized by the aggregation of water, swollen amorphous PVA containing water, and crystalline PVA which acts as a cross-linking point [1115]. The crystallinity of this gel is very low, in the range 
from $2.5 \%$ ( 1 cycle) to $6.3 \%$ (9 cycles) [13]. We have reported the coagulation size of water formed in the PVA hydrogel $[16,17]$. The coagulation diameter was estimated to be $30.2 \mathrm{~nm}$ for a $10 \mathrm{wt} \%$ gel after 2 freeze/ thaw cycles. Willcox et al. also reported the coagulation size of water with a diameter of $30 \mathrm{~nm}$ by cryogenic transmission electron microscopy (cryo-TEM) observation [18], which was in good agreement with our results [16,17].

The PVA hydrogel formed by freeze/thaw cycling has good mechanical properties. Small crystallites act as a cross-linking point to improve the mechanical properties. However, there has been no reports on the mechanical properties of PVA film prepared from a freeze/thaw cycled hydrogel. Therefore, the present work is focused on the mechanical properties of PVA film prepared by freeze/thaw cycle processes as a function of the number of freeze/thaw cycles.

\section{Experimental}

\subsection{Sample Preparation}

PVA with saponification of $98.5 \mathrm{~mol} \%$ was provided by Kuraray Co., Ltd. The stereoregularity was determined to be $\mathrm{mm}=0.23, \mathrm{mr}=0.48$, and $\mathrm{rr}=0.29$ by $1 \mathrm{H}$ nuclear magnetic resonance (NMR) measurements [16]. A PVA cast film (CF) was formed by casting the $10 \mathrm{wt} \%$ PVA solution and drying at room temperature for over a week. The film formed using the freeze/thaw cycle process was prepared as follows. $10 \mathrm{wt} \%$ PVA was dissolved in water at $120^{\circ} \mathrm{C}$ in an autoclave, and the PVA hydrogel was then formed by freezing at $-20^{\circ} \mathrm{C}$ for $5 \mathrm{~min}$ and thawing at $25^{\circ} \mathrm{C}$ for $45 \mathrm{~min}$. The number of freeze/thaw cycles employed was between 1 and 10. The gel was then dried at room temperature for over a week in a vacuum; this film is abbreviated as gel film-n (GF-n), where $n$ denotes the number of freeze/thaw cycles. The films were annealed at $130^{\circ} \mathrm{C}$ for $30 \mathrm{~min}$ in a thermostatic oven; the annealed cast and gel films are abbreviated as aCF and aGF-n, respectively.

\subsection{Measurements}

Differential scanning calorimetry (DSC) was performed on a Rigaku $8230 \mathrm{D}$ instrument at a heating rate of $5^{\circ} \mathrm{C} /$ min from room temperature to $250^{\circ} \mathrm{C}$.

The mechanical properties were measured using a tensile testing instrument (Shimadzu Instron 5566). The PVA film sample was a lateral shape with dimensions of $3.0 \times 1.0 \mathrm{~cm}^{2}$ and a thickness of $0.1-0.3 \mathrm{~nm}$. Tensile measurements were conducted with a head speed of 0.5 $\mathrm{mm} / \mathrm{min}$ for a least three film samples prepared under the same conditions, and the average value was adopted.

Dynamic mechanical analysis (DMA) was performed on a dynamic viscoelasticity instrument (I.T. Instruments
DVA-200). The heating rate was $3^{\circ} \mathrm{C} / \mathrm{min}$ in the range from -100 to $200^{\circ} \mathrm{C}$. The glass transition temperature $\left(\mathrm{T}_{\mathrm{g}}\right)$ was determined from the initial temperature required to decrease the storage modulus.

\section{Results and Discussion}

\subsection{Crystallinity of the Film Prepared by Freeze/Thaw Cycling}

Thermal analysis of the PVA film prepared by evaporating water from the freeze/thaw cycle gel was investigated to determine the crystallinity. According to a previous report, the crystallinity of the freeze/thaw cycle gel is dependent on the number of freeze/thaw cycles [13]. Therefore, the crystallinity of the GFs was investigated as a function of the number of freeze/thaw cycles after the evaporation of water. Figure 1(A) shows DSC profiles for GFs after various numbers of freeze/thaw cycles. The melting temperature was observed around $220^{\circ} \mathrm{C}$ irespective to the number of freeze/thaw cycles; however, the enthalpy for melting increased with the number of freeze/thaw cycles, which corresponds to an increase of crystallinity. Figure 2 shows the crystallinity as a function of the number of freeze/thaw cycles, where $138.6 \mathrm{~J} / \mathrm{g}$ is used for $100 \%$ crystallinity of PVA [19]. The crystallinity of the CF was as low as $22.3 \%$. A homogeneous solution of PVA/water was poured on a glass plate to form the $\mathrm{CF}$; therefore, crystallization is induced by nucleation in the homogeneous solution and subsequent

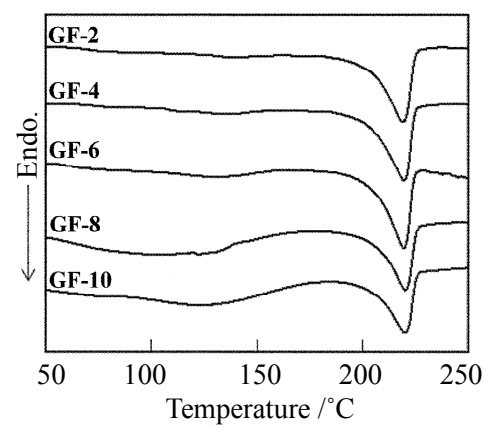

(A)

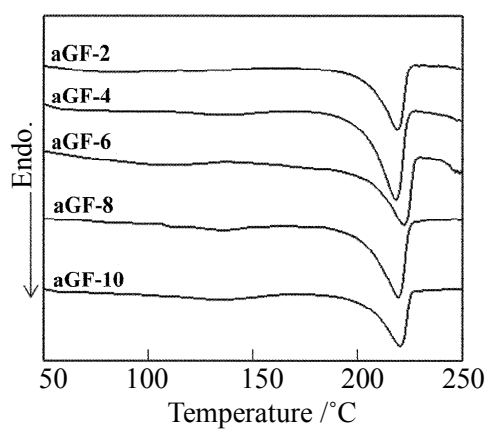

(B)

Figure 1. DSC profiles for of (A) GF and (B) aGF. 


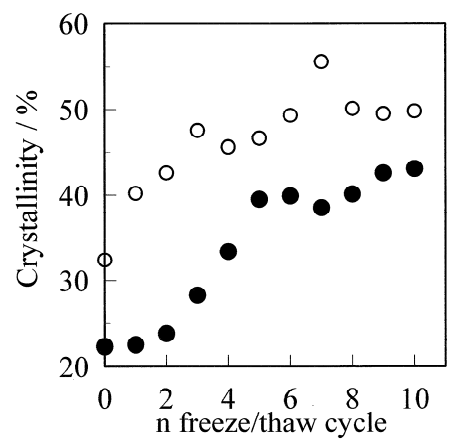

Figure 2. Crystallinity of the GF $(\bullet)$ and aGF $(\circ)$ samples as a function of the freeze/thaw cycle number. The crystallinity increased with the number of freeze/thaw cycles for both films.

crystal growth during the water evaporation process. In the case of the GF, the crystallinity increased with the number of freeze/thaw cycles. The crystallinity of GF-1 became slightly larger at $23.1 \%$, and the crystallinity of GF-10 was as high as $45.1 \%$. The crystallinity of these films is expected have a direct dependence on that of the gel prior to water evaporation, because the crystallites in the gel act as nucleation sites. Ricciardi et al. estimated the crystallinity of a hydrogel prepared by the freeze/ thaw cycle process by X-ray diffraction analysis, and reported the PVA hydrogel consists of water aggregation, swollen PVA, and crystalline PVA.

The crystallinity was increased from $2.5 \%$ ( 1 cycle) to $6.3 \%$ (9 cycles) [13]. The crystallites act as nucleators during the water evaporation process; therefore, the extent of crystallinity in the film is dependent on the number of crystallites. Thus, the main factor for crystallization in the gel formed by the freeze/thaw cycle process is considered to be the formation of small crystallites or crystal growth from small crystallites.

Hernandez et al. reported that the storage modulus of a freeze/thaw gel was increased with the number of cycles. They concluded that the number of cross-links increases with each cycle [20]. Both the increase of crystallinity and the formation of cross-links when the freeze/thaw process is repeated indicate that more crystallites would be formed in the gel, which would lead to an increase of crystallinity. During the water evaporation process, the crystallites act as nucleators to promote crystallization. This results in an increase of crystallinity for the GF after the evaporation of water with repetition of the freeze/ thaw cycles.

Crystallization is further promoted when the film is annealed. DSC profiles and the crystallinity of the annealed films are shown in Figures 1(B) and 2, respectively. The crystallinity increased after annealing. The crystallinity of the GF was larger than $40 \%$, and some of the aGF with low numbers of freeze/thaw cycles had similar crystallinity. For example, the crystallinity of
$40.2 \%$ for GF-5 is similar to that of $40.1 \%$ for aGF-1. The main factor of crystallization for the GF is crystal growth from small crystallite nucleators induced in the gel, whereas that for the annealed film is due to thermally induced crystal growth. These processes result in different crystallite sizes. The mechanical properties of these films are discussed in the following section.

\subsection{Mechanical Properties}

Figure 3(a) shows stress-strain curves for CF and GF-10. There is a significant difference between the films with and without freeze/thaw cycling treatment. For the CF sample, the maximum stress was as low as $22.3 \mathrm{MPa}$, whereas that for the GF-10 sample was $46.2 \mathrm{MPa}$, which is approximately twice as large. The maximum stresses of PVA films with similar saponification of ca. 98.5\% and additives of $\mathrm{Ag}$, aminoclay plus $\mathrm{Ag}$, and graphene have been reported to be ca. 60 [1], 72 [2], and $42 \mathrm{MPa}$ [3], respectively. Therefore, the present result is comparable to a PVA film with additives. It should be noted that the GF used in this research contains no additive. Thus, the crystallites act as a cross-linking point, which has the same role as that of the additives in References [1-3].

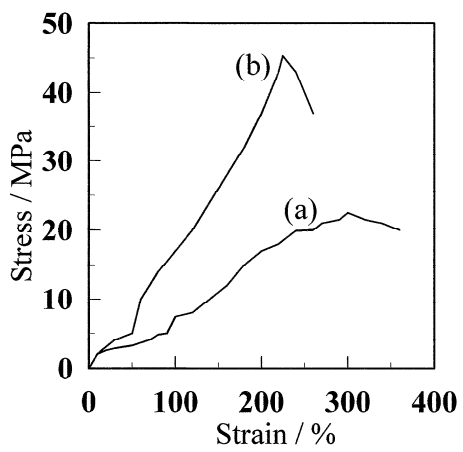

(A)

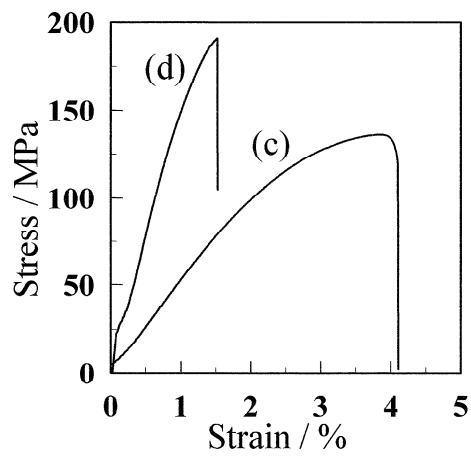

(B)

Figure 3. Stress-strain curves (A) before and (B) after annealing at $130^{\circ} \mathrm{C}$. (a) and (b) represent $\mathrm{CF}$ and GF-10, respectively. The film prepared using the freeze/thaw cycle process exhibited larger maximum stress. (c) and (d) represent aCF and aGF-10, respectively. 
The maximum stress was increased when the films were annealed at $130^{\circ} \mathrm{C}$, as shown in Figure $\mathbf{3 B}(\mathbf{b})$. The maximum stress of the aGF-10 sample was as high as 180.6 MPa, which is larger than 103.4 MPa for the aCF. The maximum stress previously reported for a cast PVA film annealed at $130^{\circ} \mathrm{C}$ was $137 \mathrm{MPa}$ [8]. Therefore, it can be concluded that the annealing of the $\mathrm{CF}$ sample is effective to increase the maximum stress of the CF sample.

The maximum stress before and after annealing is plotted as a function of the number of freeze/thaw cycles in Figure 4. The maximum stress increased with the number of freeze/thaw cycles. At over seven freeze/thaw cycled, the maximum stress became almost constant for both films before and after annealing. The maximum stress increased from 22.3 MPa for CF to 46.2 $\mathrm{MPa}$ for GF-10, which is an increase of 2.1 times, whereas that for $\mathrm{aCF}$ at $103.4 \mathrm{MPa}$ increased to $180.6 \mathrm{MPa}$ for aGF10 , which is 1.8 times larger. The increase in crystallinity is dependent on the number of freeze/thaw cycles; therefore, the maximum stress was plotted as a function of crystallinity in Figure 5. If the crystallization condition

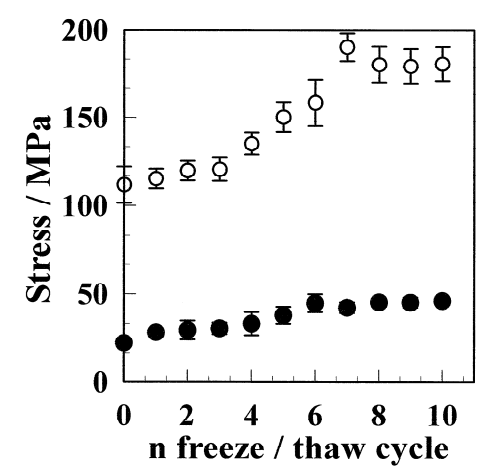

Figure 4. Maximum stress GF $(\bullet)$ and aGF $(\circ)$ depending on freeze/thaw cycle time. The maximum stress for the aGF samples was approximately three times larger than that for the GF samples.

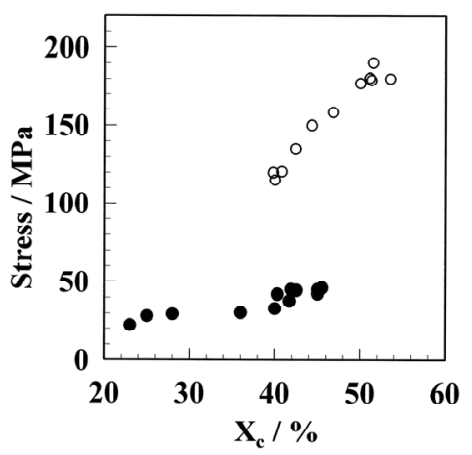

Figure 5. Relationship between the maximum stress and crystallinity for the GF $(\bullet)$ and aGF $(\circ)$ samples. Even for GF and aGF films with the same crystallinity, the maximum stress was different. Therefore, the difference in maximum stress is due to the molecular morphology. is the same, then the maximum stress can be fitted by a function. However, the dependence on crystallinity was completely different for the films before and after annealing. Therefore, the same crystallinity before and after annealing did not provide the same strain. For example, the films with ca. $40 \%$ crystallinity; the GF-1 and aGF-5 samples had maximum stress at 22.3 $\mathrm{MPa}$ and 150.2 $\mathrm{MPa}$, respectively. It can be concluded that the maximum stress of PVA films prepared under different crystallization condition is not dependent on the crystallinity; therefore, the molecular morphology should be considered. The morphological difference is attributed to the number of crystallites and the size of the crystallites. The crystallites in the GF would be small, because the film has not been annealed, and comparatively small crystallites would be dispersed in the film. In contrast, crystal growth occurs in the aGF by annealing, so that the crystallites are larger than those in the GF. This corresponds that smaller amount of crystallites provides high crystallinity. As a result, the GF requires more freeze/thaw cycles than the aGF to obtain the same crystallinity. Therefore, it was concluded that there is no direct relationship between the maximum stress and the crystallinity for GF and aGF.

Young's modulus is plotted for GF and aGF as a function of the freeze/thaw cycle in Figure 6. A similar trend to that for the maximum stress is observed, where the Young's modulus increases with the number of freeze/ thaw cycles. Young's modulus was in the range between 1.8 GPa for $\mathrm{CF}$ and 4.7 GPa for GF-10. After annealing, the modulus increased to between 2.6 for aCF and 8.9 $\mathrm{GPa}$ for aGF-10. The aGF-7 sample had the largest Young's modulus of $9.8 \mathrm{MPa}$. This is more than three times larger after freeze/thaw cycles. Konidari et al. reported that a PVA film annealed at $130^{\circ} \mathrm{C}$ has a Young's modulus of $2.26 \mathrm{GPa}$ [8]. Compared with this result, the

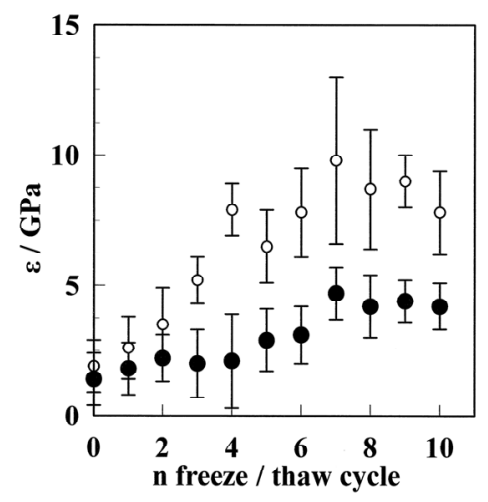

Figure 6. Young's modulus for the GF (•) and aGF (O) samples as a function of the freeze/thaw cycle number. The elastic modulus increased with the freeze/thaw cycle number for both films. The elastic modulus for aGF-6 was as high as $9.8 \mathrm{GPa}$, which is larger than that previously reported for PVA films with additives [1-3]. 
Young's modulus for aGF-7 is much larger. The present results are comparable with that reported for a PVA (saponification of 99\%)/nanodiamond (5 wt\%) composite with a Young's modulus of $10.6 \mathrm{GPa}$ [5].

The elongation at break is plotted against the number of freeze/thaw cycles in Figure 7. The CF had large elongation at break of $310 \%$. The elongation at break was slightly reduced from $290 \%$ for GF-1 to $266 \%$ for GF-10. The crystallinity of the GF is higher than that of $\mathrm{CF}$; therefore, non-crystalline chains between crystallites would be more distorted in the GF compared with the CF. This results in smaller strain for the GF. When the films are annealed, the strain is less than $10 \%$ for both cast and gel films.

The crystallinity, mechanical properties, and $\mathrm{T}_{\mathrm{g}}$ for the $\mathrm{CF}, \mathrm{GF} 10, \mathrm{aCF}$, and aGF10 samples are summarized in Table 1. The GF has a comparatively large maximum stress and Young's modulus and very large elongation at break, which corresponds to a ductile film. The aGF is characterized by very large maximum stress and Young's modulus and very small elongation at break, which corresponds to a strong film with no ductile properties.

\subsection{Glass Transition Temperature of Gel Film}

The mechanical properties are related not only to the cry-

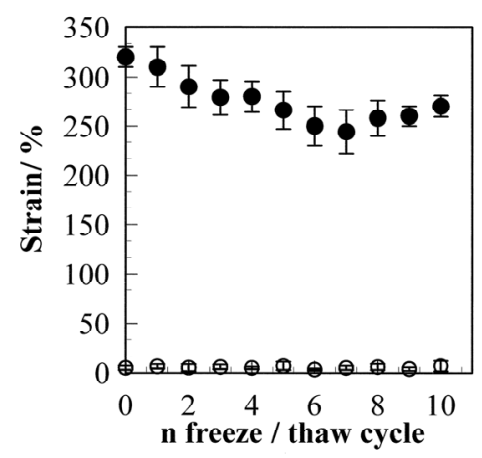

Figure 7. Elongation at break for the GF $(\bullet)$ and aGF (०) samples as a function of the freeze/thaw cycle number. The strain for the GF sample decreased with the freeze/thaw cycle number. The strain for the aGF sample was less than $10 \%$, which was much smaller than that for the GF, due to the higher crystallinity of the aGF sample.

Table 1. Crystallinity, mechanical properties, and $T_{g}$ for the CF, GF10, aCF, and aGF10 samples.

\begin{tabular}{cccccc}
\hline & Crystallinity $/ \%$ & $\sigma / \mathrm{MPa}$ & $\varepsilon / \mathrm{GPa}$ & $\Delta \mathrm{L} / \%$ & $\mathrm{Tg} /{ }^{\circ} \mathrm{C}$ \\
\hline $\mathrm{CF}$ & 22.3 & $22.3 \pm 5.3$ & $1.8 \pm 1.0$ & $310 \pm 20.0$ & 22.3 \\
$\mathrm{GF}-10$ & 43.1 & $46.2 \pm 1.9$ & $4.1 \pm 1.4$ & $266 \pm 19.2$ & 45.1 \\
$\mathrm{aCF}$ & 33.2 & $103.4 \pm 14.3$ & $2.6 \pm 1.0$ & $6.6 \pm 2.0$ & 58.6 \\
$\mathrm{aGF}-10$ & 49.8 & $180.6 \pm 9.8$ & $8.9 \pm 1.0$ & $6.3 \pm 3.7$ & 74.1 \\
\hline
\end{tabular}

$\sigma$ : maximum stress, $\varepsilon$ : Young's modulus, and $\Delta \mathrm{L}$ : elongation at break. stallinity, but also the molecular structure of amorphous. Therefore, $T_{g}$ was measured using DMA. Figure 8 shows the dependence of the storage modulus on the temperature for $\mathrm{CF}$ and $\mathrm{GF}$ before and after annealing at $130^{\circ} \mathrm{C}$. The $\mathrm{T}_{\mathrm{g}}$ of $\mathrm{CF}$ was observed at $22.3^{\circ} \mathrm{C}$, whereas that for the GF- 10 was $42.8^{\circ} \mathrm{C}$. A comparatively large number of small crystallites are formed in the GF. The amorphous chain between crystallites would be more distorted than that for $\mathrm{CF}$, so that the $\mathrm{T}_{\mathrm{g}}$ is higher. When the films are annealed, the $\mathrm{T}_{\mathrm{g}}$ increases for both $\mathrm{CF}$ and $\mathrm{GF}$. The crystal growth due to annealing makes the amorphous region small, and the amorphous chain would be more distorted, which results in higher $\mathrm{T}_{\mathrm{g}}$.

Figure 9 shows $T_{g}$ as a function of the number of freeze/thaw cycles. $T_{\mathrm{g}}$ increases with the number of freeze/thaw cycles for both GF and aGF. The $\mathrm{T}_{\mathrm{g}}$ of aGF increased from $45.3^{\circ} \mathrm{C}$ for aGF- 1 to $72.1^{\circ} \mathrm{C}$ for aGF-10, in contrast to the increase from $27.6^{\circ} \mathrm{C}$ to $45.2^{\circ} \mathrm{C}$ for the non-annealed GF. This can be explained by the increase in distorted non-crystalline chains between crystallites with annealing.

The relationship between the maximum stress and $T_{g}$ was investigated. Figure $\mathbf{1 0}$ shows the maximum stress as a function of $T_{g}$. In contrast to the relationship between the maximum stress and the crystallinity shown in Figure 5, the experimental result can be fitted by the following equation:

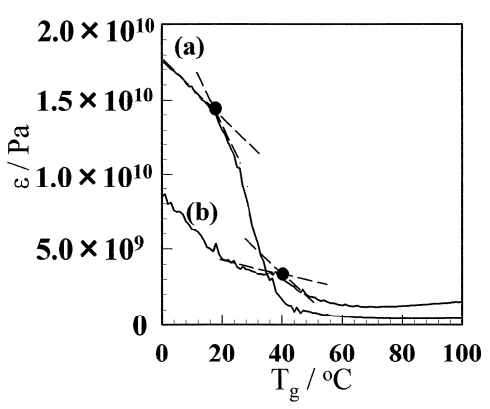

(A)

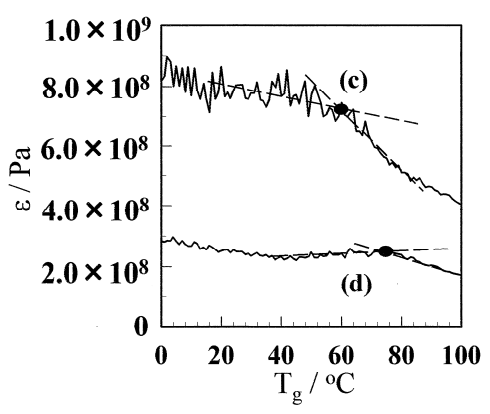

(B)

Figure 8. Dynamical mechanical analysis results for the (A) GF and (B) aGF samples. (a) and (b) represent CF and GF10, respectively. The film prepared using the freeze/thaw cycle process exhibited higher $\mathrm{Tg}$. (c) and (d) represent $\mathrm{aCF}$ and aGF-10, respectively. 


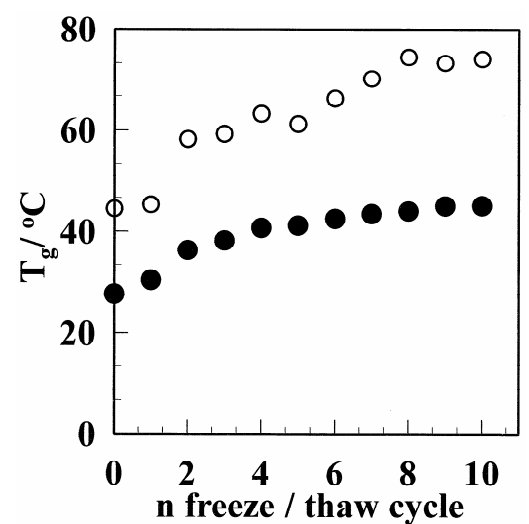

Figure 9. $T_{g}$ for the GF $(\bullet)$ and aGF $(\circ)$ films as a function of the freeze/thaw cycle number. $T_{g}$ increased with the freeze/thaw cycle number and was higher for the aGF sample than the GF sample.

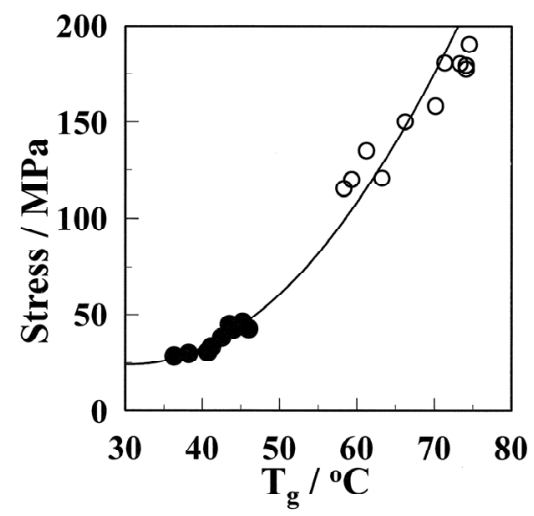

Figure 10. Relationship between the maximum stress and $T_{g}$ for the GF $(\bullet)$ and aGF $(\circ)$ samples.

$$
\mathrm{S}_{\max }=0.050 \mathrm{~T}_{\mathrm{g}}^{2}+1.2 \mathrm{~T}_{\mathrm{g}}
$$

where $S_{\max }$ denotes the maximum stress. Therefore, the maximum stress can be expressed as a function of $T_{g}$. The mobile component of the amorphous chain, which results in low $\mathrm{T}_{\mathrm{g}}$, would be broken more easily than the hard one. Therefore, it is concluded that the important factor that determines the maximum stress is $T_{g}$. Thus, high $T_{g}$, which corresponds to less mobile amorphous chains, results in an increase of the maximum stress.

\section{Conclusions}

The mechanical properties of PVA films prepared from freeze/thaw cycled gel were investigated. During the water evaporation process, crystal growth was promoted by the small crystallites formed in the gel during the freeze/ thaw cycle process. The crystallinity of the GF increased with the number of freeze/thaw cycles, which corresponded to the formation of more crystallites. The maximum stress of the GF was larger than that of the CF and increased with the number of freeze/thaw cycles. The largest maximum stress of GF was 46.2 MPa for GF-10, which was approximately twice that of $22.3 \mathrm{MPa}$ for the $\mathrm{CF}$. No additives were used in these films, but the maximum stress of the GF was comparable with previously reported PVA films containing additives. Annealing of the films at $130^{\circ} \mathrm{C}$ resulted in an increase of the maximum stress. The maximum stress of $46.2 \mathrm{MPa}$ for GF-10 became 180.6 MPa for aGF-10. The Young's modulus was as high as $9.8 \mathrm{GPa}$ for aGF-7, which was three times larger than that for $\mathrm{aCF}$ at $2.6 \mathrm{GPa}$. The elongation at break for GF was almost comparable to that for $\mathrm{CF}$, and it remained small for $\mathrm{aCF}$ and aGF after annealing. In conclusion, GF was stronger and more ductile than $\mathrm{CF}$, and the aGF was much stronger with small strain.

The maximum stress was not well correlated with the crystallinity for GF and aGF, but was fitted as a function of $T_{g}$ using Equation (1), where high $T_{g}$ corresponded to rigid amorphous chain between crystallites that resulted in large maximum stress. This can be explained by the molecular morphology. The non-annealed GF consists of small crystallites, but the aGF film consists of comparatively large crystallites promoted by the annealing process. Crystal growth allows the amorphous chains to become more distorted.

\section{Acknowledgements}

The authors would like to thank Dr. Ohgi of Kuraray Co., Ltd., for kindly providing the PVA sample.

\section{REFERENCES}

[1] H. Mbhele, M. G. Salemane, C. G. C. van Sittert, J. M. Nedeljkovic, V. Djokovic and A. S. Luyt, "Study of Sago Starch-CdS Nanocomposite Films: Fabrication, Structure, Optical and Thermal Properties," Chemistry of Materials, Vol. 15, No. 26, 2003, pp. 5019-5024. http://dx.doi.org/10.1021/cm034505a

[2] G. Johnsy, K. K. R. Datta, V. A. Sajeevkumar, S. N. Sabapathy, A. S. Bawa and M. Eswaramoorthy, "Direct Assembly of Photoresponsive C60-Gold Nanoparticle Hybrid Films," Applied Materials \& Interfaces, Vol. 1, No. 12, 2009, pp. 2796-2803. http://dx.doi.org/10.1021/am9005226

[3] X. Zhao, Q. Zhang and D. Chen, "Direct Assembly of Photoresponsive C60-Gold Nanoparticle Hybrid Films," Macromolecules, Vol. 43, No. 5, 2010, pp. 2357-2363. http://dx.doi.org/10.1021/ma902862u

[4] X. Zhang, T. Liu, T. V. Sreekumur, S. Kumar, V. C. Moore and R. H. Hauge, "Smalley Poly(vinyl alcohol)/SWNT Composite Film E," Nano Letters, Vol. 3, No. 9, 2003, pp. 1285-1288. http://dx.doi.org/10.1021/n1034336t

[5] S. Morimune, M. Kotera, T. Nishino, K. Goto and K. Hata, "Poly(vinyl alcohol)/Graphene Oxide Nanocomposites Prepared by a Simple Eco-Process," Macromolecules, Vol. 44, No. 11, 2011, pp. 4415-4421. http://dx.doi.org/10.1021/ma200176r 
[6] P. Podsiadlo, A. K. Kaushik, B. S. Shim, A. Agarwal, Z. Tang, A. M. Waas and E. M. J. Arruda, "Can Nature's Design Be Improved upon High Strength, Transparent Nacre-Like Nanocomposites with Double Network of Sacrificial Cross Links," The Journal of Physical Chemistry B, Vol. 112, No. 46, 2008, pp. 14359-14363. http://dx.doi.org/10.1021/jp801492n

[7] K. E. Prasad, B. Das, U. Maitra, U. Ramamurty and C. N. R. Rao, "Extraordinary Synergy in the Mechanical Properties of Polymer Matrix Composites Reinforced with 2 Nanocarbons," Proceedings of the National Academy of Sciences of the USA, Vol. 106, No. 32, 2009, pp. 1318613189. http://dx.doi.org/10.1073/pnas.0905844106

[8] M. V. Konidari, K. G. Papadokostaki and M. Sanopoulou, "Moisture-Induced Effects on the Tensile Mechanical Properties and Glass-Transition Temperature of Poly(vinyl alcohol) Films," Journal of Applied Polymer Science, Vol. 120, No. 6, 2011, pp. 3381-3386. http://dx.doi.org/10.1002/app.33118

[9] N. A. Peppas, "Turbidimetric Studies of Aqueous Poly(vinyl alcohol) Solutions," Macromolecular Chemistry and Physics, Vol. 176, No. 11, 1975, pp. 3433-3440. http://dx.doi.org/10.1002/macp.1975.021761125

[10] C. M. Hassan and N. A. Peppas, "Structure and Applications of Poly(vinyl alcohol) Hydrogels Produced by Conventional Crosslinking or by Freezing/Thawing Methods," Advances in Polymer Science, Vol. 153, 2000, pp. 37-65. http://dx.doi.org/10.1007/3-540-46414-X_2

[11] V. I. Lozinsky, "Cryotropic Gelation of Poly(vinyl alcohol) Solutions," Russian Chemical Reviews, Vol. 67, No. 7, 1998, pp. 573-586. http://dx.doi.org/10.1070/RC1998v067n07ABEH000399

[12] V. I. Lozinsky, I. Y. Galaev, F. M. Plieva, I. N. Savina, H. Jungvid and B. Mattiasson, "Polymeric Cryogels as Promising Materials of Biotechnological Interest," Trends in Biotechnology, Vol. 21, No. 10, 2003, pp. 445-451. http://dx.doi.org/10.1016/j.tibtech.2003.08.002

[13] R. Ricciawdi, F. Auriemma, C. De Rosa and F. Laupretre, "X-Ray Diffraction Analysis of Poly(vinyl alcohol) Hydrogels, Obtained by Freezing and Thawing Techniques," Macromolecules, Vol. 37, No. 5, 2004, pp. 1921-1927. http://dx.doi.org/10.1021/ma035663q

[14] R. Ricciardi, F. Auriemma, C. Gaillet, C. De Rosa and F. Laupretre, "Investigation of the Crystallinity of Freeze/ Thaw Poly(vinyl alcohol) Hydrogels by Different Techniques," Macromolecules, Vol. 37, No. 25, 2004, pp. 9510 9516. http://dx.doi.org/10.1021/ma048418v

[15] R. Ricciardi, G. Mangiapia, F. Lo Celso, L. Paduano, R. Triolo, F. Auriemma, C. De Rosa and F. Laupretre, "Evolution of PVA Gels Prepared without Crosslinking Agents as a Cell Adhesive Surface," Chemistry of Materials, Vol. 17, No. 5, 2005, pp. 1183-1189. http://dx.doi.org/10.1021/cm048632y

[16] T. Nakaoki and H. Yamashita, "Coagulation Dimension of Freezable Bound Solvent in Isotactic Polypropylene/oDichlorobenzene Gel," Journal of Molecular Structure, Vol. 875, No. 1-3, 2008, pp. 282-287. http://dx.doi.org/10.1016/j.molstruc.2007.04.040

[17] T. Nakano and T. Nakaoki, "Coagulation Size of Freezable Water in Poly(vinyl alcohol) Hydrogels Formed by Different Freeze/Thaw Cycle Periods," Polymer Journal, Vol. 43, 2011, pp. 875-880. http://dx.doi.org/10.1038/pj.2011.92

[18] P. J. Willcox, D. W. Howie, K. Shimidt-Rohr, D. A. Hoagland, S. P. Gido, S. Pudjijanto, L. W. Kleiner and S. J. Venkatraman, "Microstructure of Poly(vinyl alcohol) Hydrogels Produced by Freeze/Thaw Cycling," Journal of Polymer Science Part B, Vol. 37, No. 24, 1999, pp. 34383454.

http://dx.doi.org/10.1002/(SICI)1099-0488(19991215)37: 24<3438::AID-POLB6>3.0.CO;2-9

[19] A. S. Hickey and N. A. Peppas, "Mesh Size and Diffusive Characteristics of Semicrystalline Poly(vinyl alcohol) Membranes Prepared by Freezing/Thawing Techniques," Journal of Membrane Science, Vol. 107, No. 3, 1995, pp. 229 237. http://dx.doi.org/10.1016/0376-7388(95)00119-0

[20] R. Hernandez, A. Sarafian, D. Lopez and C. Mijangos, "Structure of Poly(vinyl alcohol) Cryo-Hydrogels as Studied by Proton Low-Field NMR Spectroscopy," Polymer, Vol. 46, 2004, pp. 5543-5550. 